\title{
Quality improvement project: outcomes before and after routine orthogeriatric review of all trauma admissions aged 65 and over
}

\author{
Author: Amy Davies
}

\begin{abstract}
Aims
The importance of comprehensive geriatric assessment (CGA) is well-recognised among patients with neck of femur fractures (NOF\#). Current practice locally involves patients with NOF\# being admitted under joint orthopaedic/orthogeriatric care, then continuing under orthogeriatrics postoperatively..

In contrast, patients with non-NOF\# remain under orthopaedic care throughout admission. Subsequently orthogeriatric input is reactive to acute deterioration, rather than preventative. This prompted a quality improvement project to investigate the impact of regular orthogeriatric review on the care of all elderly orthopaedic patients.
\end{abstract}

\section{Methods}

An initial 4-week scoping exercise looked at the number of trauma admissions aged $>65$. Over the subsequent 4 weeks, patients aged $>65$ admitted with trauma (excluding NOF\#) were identified at the daily trauma meeting. They were admitted under an orthopaedic consultant and seen daily as per standard care.

Each patient received a CGA on admission and weekly reviews by the orthogeriatricians. Unwell patients were seen more frequently. A multidisciplinary meeting was held during the pilot.

\section{Results}

Fifty patients aged $>65$ years were admitted with trauma. Mean age was 79 (65-94 years), 62\% female. 104 patient reviews were completed.

Seventy-eight per cent sustained injuries due to a fall or collapse. $54 \%$ had fractures of which $48 \%$ were fragility fractures. $25 \%$ had sustained an intracranial bleed.

Fifty per cent had analgesia increased and $34 \%$ had laxatives increased. $22 \%$ had reduction in polypharmacy and $14 \%$ had new medication commenced (excluding analgesia or laxatives).

Twenty per cent had vitamin D deficiency treated. $75 \%$ of patients with fragility fractures had a bone health assessment. $16 \%$ of patients had advanced care planning (ACP) discussions and
$10 \%$ involved completion of a Recommended Summary Plan for Emergency Care and Treatment (RESPECT) form.

\section{Conclusion}

Elderly patients commonly suffer falls and have complex comorbidities. Early CGA allows interventions including optimisation of medication, bone plan commencement and early advance care planning. Expansion of regular orthogeriatric care throughout all orthopaedic admissions would result in a significant workload with staffing implications. The cost of this is likely to be offset by the significant improvement to patient care and reduction in medical complications.

\section{Conflict of interest statement}

No conflict of interests declared. 